\title{
Stepwise evolution of molecular nanoaggregates inside the pores of a highly flexible Metal-Organic Framework
}

\author{
P. P. Mazzeo ${ }^{1,2}$, D. Balestri ${ }^{1}$, C. Carraro', N. Demitri ${ }^{3}$, P. Pelagatti ${ }^{1,4}$, A. Bacchi ${ }^{1,2}$ \\ ${ }^{I}$ Dipartimento di Scienze Chimiche, della Vita e della Sostenibilità Ambientale, Università di Parma, Viale delle Scienze, 17A, 43124 \\ Parma (Italy), ${ }^{2}$ Biopharmanet-TEC, Università degli studi di Parma via Parco Area delle Scienze 27/A, 43124 Parma (Italy), \\ ${ }^{3}$ Elettra-Sincrotrone Trieste S.S. 14 Km 163.5 in Area Science Park, 34149 Basovizza-Trieste (Italy), ${ }^{4}$ Centro Interuniveristario \\ di Reattività Chimica e Catalisi (CIRCC) Via Celso Ulpiani 27, 70126 Bari (Italy)
}

\section{paolopio.mazzeo@unipr.it}

Hollow molecular structures capable of guest inclusion represent an area of raising interest and lie at the forefront of the modern supramolecular chemistry.[1,2] Originally studied in solution, this concept has been extended in the solid state, after the pioneer work on the "crystalline sponge method" (CSM). [3] The CSM primary application has been the unambiguous structural determination via SC-XRD of a single analyte encapsulated inside a porous MOF. However, as the host-guest systems often show severe disorder, their reliable crystallographic determination is very demanding [1,2] thus the dynamics of the guest entering and the formation of nanoconfined molecular aggregates has not been in the spotlight yet.

We extended the concept of the CSM stepwisely monitoring the structural evolution of nanoconfined supramolecular aggregates of guest molecules with the concomitant displacement of pristine DMF inside the cavities of a novel flexible MOF, PUM168. Furthermore, we correlated this phenomenon to the structural reorganization of the host framework, elucidating the dynamic interplay between the container and the content. [4] In order to deeply understand the "physiology" of PUM168 breathing during the guest uptake, we focused our attention on the three main actors involved in the play: i) the MOF structure, ii) the leaving DMF molecules trapped during the synthesis of the MOF and iii) the incoming guest molecules uptaken during the soaking process. [5]

The fate of each actor influences and is influenced by the other two characters, in a play that shows how the structure of the framework changes in response of the guest positioning and composition. [5]
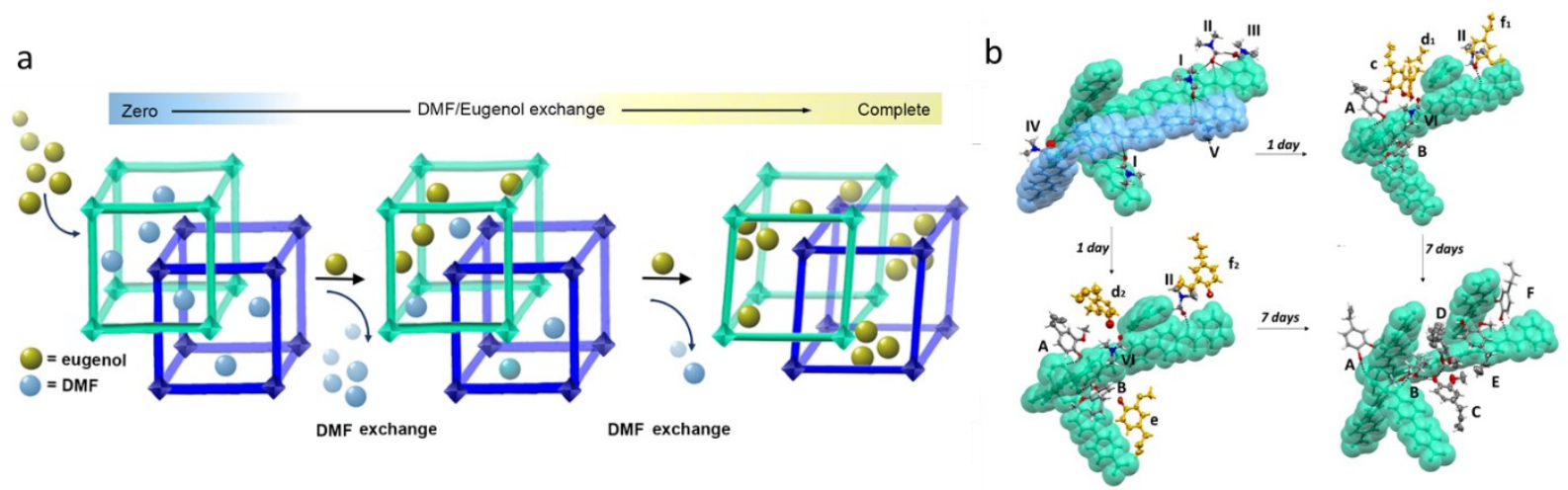

Figure 1. a) schematic representation of the PUM168 solvent-to-guest exchange. b) After 1-day of soaking DMF is partially replaced by guest, after 7 days PUM168 nets rearrange and guests organize in a supramolecular cluster.

[1] K. Rissanen, Chem. Soc. Rev 2017, 46, 2638.

[2] S. Lee, H.-B. Bu, S. A. Alshmimri, O. M. Yaghi, J. Am. Chem. Soc. 2018, 140, 8958-8964.

[3] Y. Inokuma, S. Yoshioka, J. Ariyoshi, T. Arai, Y. Hitora, K. Takada, S. Matsunaga, K. Rissanen, M. Fujita, Nature 2013, 495, $461-466$.

[4] M. Shivanna, Q. Yang, A. Bajpai, M. J. Zaworotko, E. Patyk-kazmierczak, Nat. Commun. 2018, 9, 1-7.

[5] D. Balestri, P. P. Mazzeo, C. Carraro, N. Demitri, P. Pelagatti, A. Bacchi, Angew. Chem. Int. Ed. 2019, 58, 17342-17350.

Keywords: crystalline sponge method; MOFs; nanoaggregates; molecular motion; crystal-to-crystal investigation 